\title{
The efficacy of computer-assisted cognitive behavioral therapy (cCBT) on psychobiological responses and perioperative outcomes in patients undergoing functional endoscopic sinus surgery: a randomized controlled trial
}

Yang Yang ${ }^{1,2}$, Yuling $\mathrm{Li}^{2}$, Haibin Zhang ${ }^{3}$, Yong $\mathrm{Xu}^{4,5^{*}}$ and Binquan Wang ${ }^{6,7^{*}}$

\begin{abstract}
Background: Functional endoscopic sinus surgery (FESS) is required to minimize bleeding to maintain a clear operative field during surgery, so it is important to preoperative anti-anxiety and stable hemodynamics. Initial evidence suggests cognitive behavioral therapy (CBT) is effective to minimize surgery-related stress and to speed up recovery. The study aimed to evaluate the efficacy of a newly developed computer-assisted CBT (cCBT) program on surgery-related psychobiological responses in patients undergoing FESS.

Methods: Participants were allocated to a CCBT group ( $(C B T ; n=50$ ) or a UC group (usual care; $n=50$ ) by random number table. The State Anxiety Inventory (SAI), Patients Health Questionnaire-9 (PHQ-9), Athens Insomnia Scale (AIS), systolic blood pressure (SBP), diastolic blood pressure (DBP), and heart rate (HR) were assessed before intervention (T1), at $1 \mathrm{~h}$ before operation (T2), at postoperative $48 \mathrm{~h}$ (T3), and $96 \mathrm{~h}$ (T4: after intervention completed) respectively. The stress hormone was assayed at T1 and T2. The duration of surgery, anesthesia, and post-anesthesia care unit (PACU) were recorded. A satisfaction survey about nursing services was completed by participants before discharge.

Results: Compared to the UC group, the SAI scores at T2 and the AIS scores at T3 were lower in the CCBT group ( $p$ $<0.01$ and $p=0.002)$. The positive rate of participants who were moderate and severe anxiety (SAI score $>37)$ at T2 were lower (72\% vs. 88\%, $p=0.04)$; the cortisol levels, SBP, DBP, and HR at T2 in the CCBT group were lower $(p=0.019$ and all $p<0.01)$; the duration of anesthesia and PACU was shorter $(p=0.001$ and $p<0.01)$; the CCBT group showed higher satisfaction scores.
\end{abstract}

\footnotetext{
* Correspondence: xuyongsmu@vip.163.com; wbq_xy@sxent.org

${ }^{4}$ Department of Psychiatry, The First Hospital of Shanxi Medical University, Taiyuan, People's Republic of China

${ }^{6}$ Department of Otorhinolaryngology Head and Neck Surgery, The First Hospital of Shanxi Medical University, Taiyuan, People's Republic of China

Full list of author information is available at the end of the article
}

C C The Author(s). 2021 Open Access This article is licensed under a Creative Commons Attribution 4.0 International License, which permits use, sharing, adaptation, distribution and reproduction in any medium or format, as long as you give appropriate credit to the original author(s) and the source, provide a link to the Creative Commons licence, and indicate if changes were made. The images or other third party material in this article are included in the article's Creative Commons licence, unless indicated otherwise in a credit line to the material. If material is not included in the article's Creative Commons licence and your intended use is not permitted by statutory regulation or exceeds the permitted use, you will need to obtain permission directly from the copyright holder. To view a copy of this licence, visit http://creativecommons.org/licenses/by/4.0/ The Creative Commons Public Domain Dedication waiver (http://creativecommons.org/publicdomain/zero/1.0/) applies to the data made available in this article, unless otherwise stated in a credit line to the data. 
Conclusion: The newly developed CCBT program was an effective non-pharmacological adjunctive treatment for improving the surgery-related psychosomatic responses and perioperative outcomes.

Trial registration: The study was registered with the Chinese Clinical Trial Registry (ChiCTR1900025994) on 17 September 2019.

Keywords: Computer-assisted cognitive behavioral therapy, Psychobiological response, Functional endoscopic sinus surgery, Anxiety, Insomnia, Cortisol

\section{Background}

Chronic rhinosinusitis (CRS) is a chronic local inflammatory disease with a relatively high incidence. The overall prevalence of CRS was $8.0 \%$ in seven cities in mainland China (Shi et al. 2015). Once CRS becomes refractory and no longer responsive to medication, functional endoscopic sinus surgery (FESS) represents the first choice for surgical treatment (Kaper et al. 2020). Minimizing blood loss and achieving the optimal intraoperative surgical field visibility are two important determinants of surgical success and outcome in FESS (Chhabra et al. 2020). Perioperative hemodynamic management can optimize surgical conditions, minimize bleeding, and optimize patient outcomes in FESS (Martin et al. 2020). Preoperative anxiety influences physiological outputs including increased heart rate, decreased heart rate variability, blunted post-stress systolic blood pressure recovery, and elevated levels of cortisol (Epel, 2020, Landon et al. 2019).

The studies on controlled hypotension and reducing intraoperative bleeding are mainly focused on the comparison of total intravenous anesthesia (TIVA) and inhalational anesthesia (IA) (Lu et al. 2020) and the use of drugs (Chhabra et al. 2020). To minimize the adverse reaction, using properly non-invasive and nonpharmaceutical healing techniques may be an additional approach. And the perioperative psychological interventions based on multidisciplinary therapy have proved to be an effective and adequate strategy (Visioni et al. 2018). The cognitive-behavioral therapy in psychological interventions was proved to influence positively on modulating the surgical stress response and improving surgical outcomes, particularly in those with maladaptive features (Villa et al. 2020).

Cognitive-behavioral therapy (CBT) is the first choice recommended by the FDA for the non-pharmacologic treatment of depression and anxiety disorders, which focuses on correcting maladaptive behavior and negative thoughts (Beck, 1964). CBT had been proved feasible and acceptable to improve symptoms of depression or anxiety, health-related quality of life (HRQOL) in patients undergoing surgery (Dao et al. 2011). Multiple barriers prevent patients undergoing major surgery from accessing the CBT, such as a shortage of trained therapists, high costs, and a lack of accessibility in remote areas, which contribute to lacking patient engagement (Cartreine et al. 2010). The computer-assisted cognitive behavioral therapy (cCBT) had been developed as effective psychotherapy and reveals promise for overcoming barriers in medical settings. cCBT has been suggested as an attractive alternative treatment to faceto-face treatment, which is a remote mental-health intervention model and presents structured sessions of CBT via a computer interface or smartphone application (Carlbring et al. 2018). The overall results of RCT suggest that CCBT can be an effective treatment for depression and health anxiety in primary care (Andrews et al. 2018). Moreover, it can be a useful adjunct treatment for somatic conditions with anxiety and depression (Wright et al. 2018). However, the implementation of cCBT in ameliorating negative emotions and somatic distress associated with surgery is limited. There are few study reports on $\mathrm{CCBT}$ intervention in patients with FESS.

Accordingly, the current study aimed to conduct an RCT to test the efficacy of a newly developed CCBT program on improving psychological and physical parameters compared to a matched control condition. The hypotheses of the study are as follows: the patients of the CCBT group will express more satisfying subjective perception and show objective indications in favor of treatment and rehabilitation than the control group, such as less preoperative anxiety, postoperative depression, insomnia, cortisol, and more stable hemodynamics (BP and HR).

\section{Methods}

\section{Study design}

The present study was a prospective, randomized, controlled, and single-blind trial with one experimental arm (CCBT group) and one control arm (UC group). Participants were allocated one of two groups according to random number table: either five sessions of CCBT or health education based on usual care. Participants were recruited from the Otolaryngology Department through the First Hospital of Shanxi Medical University from 20 September 2019 to 10 January 2020. The patient, attending anesthesiologist and surgeon were allocated blindly to the study groups. Surgeons were made up of two teams 
which were allocated task randomly. Anesthesiologist is a specialized doctor whose clinical working time is more than 10 years and responsible for the anesthesia of all participants.

\section{Anesthesia technique}

The anesthesia way was TIVA with routine paralysis and endotracheal intubation. Anesthesia was induced with midazolam $(0.03 \mathrm{mg} / \mathrm{kg})$, etomidate $(0.3 \mathrm{mg} / \mathrm{kg})$, sufentanil $(0.5 \mu \mathrm{g} / \mathrm{kg})$, and cisatracurium $(0.15 \mathrm{mg} / \mathrm{kg})$ to facilitate oral intubation. The anesthesia was maintained with a continuous infusion of propofol infusion $(4 \sim 6 \mathrm{mg} /$ $\mathrm{kg} / \mathrm{h})$ and remifentanil $(0.1 \sim 0.3 \mu \mathrm{g} / \mathrm{kg} / \mathrm{min})$ by a targetcontrolled infusion (TCI) to maintain bispectral index (BIS) at 40 to 60. A controlled hypotension was provided and adjusted according inter-operative bleeding (target: SBP up to $80 \sim 90 \mathrm{mmHg}$, arterial pressure up to $50 \sim$ $70 \mathrm{mmHg}$, or a decrease up to $30 \%$ than mean arterial pressure) (Modir et al. 2018). The patient was transferred from the PACU to the general ward, if Steward score was assessed $>=4$ (or the modified Aldrete score was $>=9$ ) (Sun et al. 2017).

\section{Surgical technique}

After induction of general anesthesia, local anesthesia and epinephrine 1:1000 soaked cotton wool pledgets were applied the nasal cavity for 5 to $10 \mathrm{~min}$ to reduce bleeding. The surgical technique consisted of bilateral anterior and posterior ethmoidectomy with bilateral middle meatotomy according to Messerklinger technique developed by Professor Walter Messerklinger, which only the Ostiomeatal Complex (OMC) lesions were removed without damage to the mucosa in the sinuses(Kane, 2020).

\section{Participants}

Inclusion criteria were the following: (1) aged $18 \sim 60$ years; (2) scheduled to undergo FESS within a week; (3) general anesthesia can be acceptable as a form of anesthesia; (4) American Society of Anesthesiologists (ASA) grades I and II; (5) be willing to answer the questionnaires and participate in the interventions. Exclusion criteria included the following: (1) diagnosis of definite history of psychiatric illness, substance abuse/dependence within 12 months before enrollment; (2) undergoing a surgery that was postponed for more than a week or changed to emergent surgery; (3) currently receiving any psychiatric or psychological treatment, including psychiatric medication; (4) the Patient Health Questionnaire Depression Scale-9 (PHQ-9) score $\geq 20$ or the risk of suicide (5) those who had serious cardiovascular and respiratory diseases.

\section{Measures and outcomes}

Participants were identified and confirmed from surgical-plan lists by the Electronic Medical Record System. Then, they were approached by researchers and written informed consent would be obtained according to the ethical requirements before any study procedure. The participants who met the inclusion criteria were enrolled and assessed to collect demographic information and baseline data. Two study staff were blinded to collect and analyze data separately.

Baseline characteristics included sex, age, education, principal diagnosis, surgery type, and anesthesia grading. The baseline measures (anxiety, depression, insomnia, stress hormones, vital signs) were collected on the day after hospitalization (before intervention: T1). The state anxiety, stress hormones, and vital signs were reobtained from patients $1 \mathrm{~h}$ before surgery (T2). The depression, insomnia, and vital signs were re-assessed at postoperative $48 \mathrm{~h}$ (T3). After all interventions are completed, anxiety, depression, insomnia, and vital signs were re-assessed at postoperative $96 \mathrm{~h}$ (T4). And a satisfaction survey about nursing services was completed by participants before discharge. The duration of surgery, anesthesia, and post-anesthesia care unit (PACU) were recorded.

State-Trait Anxiety Inventory (STAI) was developed by Spielberger C.D and Gorsuch R.L (Spielberger et al. 1983). State Anxiety Inventory (SAI) assesses current state of anxiety at a particular moment and Trait Anxiety Inventory (TAI) evaluates relatively stable aspects of "anxiety proneness" (Spielberger et al. 1983). These scale were reliable, and Cronbach's alpha $=0.80$ and 0.85 for SAI and TAI for the current sample. Past studies displayed that SAI score $>37$ was considered to be moderate and severe anxiety (Pan et al., 2018).

The Patient Health Questionnaire Depression Scale-9 (PHQ-9) can establish grade depressive symptom severity (Kroenke et al. 2001). We obtained a value of Cronbach's $\alpha$ was 0.80 for the current sample. Athens Insomnia Scale (AIS) was developed by Soldatos to quantify sleep difficulty based on ICD-10 criteria (Soldatos et al. 2000). Cronbach's $\alpha$ was 0.90 for the current sample. The PHQ-9 score $>4$ and the AIS score $>5$ were considered a depression or insomnia clinical cutoff state (Kroenke et al. 2001, Soldatos et al. 2003).

We collected venous blood of a part of participants (60/100) between 6:00 a.m. and 8:00 a.m. to assay serum cortisol and Adrenocorticotropic Hormone (ACTH) at $\mathrm{T} 1$ and T2 respectively. A single venipuncture collected $5 \sim 8 \mathrm{ml}$ blood into purple and yellow plain tubes (with coagulants and separation gel). The cortisol and ACTH levels were measured by chemiluminescence assay (CLIA) and microparticles CLIA. 


\section{Study interventions Introduction}

The potential participants were given a brief introduction about the study program before beginning the fivesession intervention, especially the importance of mental health for postoperative recovery. Eligible in-patients who were willing to participate were registered and kept in touch by study staff.

\section{CCBT program intervention}

The new cCBT program named "computer-assisted psychosomatic cognitive behavioral therapy during perioperative period (CPCBT-Period)" was developed and optimized based on Enhanced Recovery After Surgery (ERAS), nursing education and CBT theory. The CCBT group received the optimized $\mathrm{CCBT}$ intervention in addition to usual care. The cCBT is a non-internetbased computer program that includes registration and therapy conducted in a medical setting. The program is based on condition-surgery and CBT elements, which comprised five sessions, and each session took about 20 min to complete. The preoperative preparation time is usually only $3( \pm 1)$ days for patients with FESS since admission and postoperative recovery time is about $7( \pm 2)$ days. The time points of intervention are 2 days and 1 day before surgery and 2 days, 3 days, and 4 days after surgery. All sessions would finish before discharge. Participants in the CCBT group entered their information into the program and the first treatment was administered soon after registration. Each session starts by logging into the admission number. Next time logging in, individualized treatment will continue. The treatment modules within the program included cognitive therapy, cognitive consolidation, and behavioral relaxation therapy. The contents of perioperative education were reviewed and passed by clinical nursing specialists which were unique and customized for surgical patients. The primary components of the cognitive therapy module included the following:

Session 1: Preoperative psychological preparation;

Session 2: Preoperative physical preparation and introduction to the surgical environment;

Session 3: Management of postoperative pain, insomnia, and anti-thrombus;

Session 4: Postoperative exercise and diet;

Session 5: Education on steps following hospital discharge.

The behavioral therapy module included relaxing training, such as imaginative relaxing exercise, progressive muscle relaxation, breathing exercises, relaxing sleep exercise, and a mindfulness meditation body scan. Homework as cognitive consolidation was then provided for participants to answer questions in the form of a game, in which the questions served as a review of the previous cognitive therapy module. The cognitive therapy module and behavioral therapy module were presented via video. The contents and design were reviewed and passed by psychotherapists.

\section{Usual care intervention}

In the UC group, patients were administered the FESS routine care conducted according to the Perioperative Care Manual and Consensus on ERAS (Chen Bing, 2018). To match the CCBT group, participants randomized to this condition, including five sessions, focused on education about the illness, surgery, anesthesia, and postoperative nursing. These sessions were developed in consultation with clinical nursing specialists. A 20-min verbal briefing per session was administered to participants by study staff. Topics included the introduction of illness and surgery; preoperative psychological and physical preparation; postoperative disease care; activities and diet after surgery; and education on steps following hospital discharge.

\section{Data analysis}

To have a difference greater than 5 in average levels of state anxiety between groups used PASS11.0, we estimated a sample size of 46 patients per arm according to the previous value of the standard deviation $\sigma=10$ (Ruffinengo et al. 2009). Allowing for a 10\% dropout rate, approximately 50 cases were ultimately included per group.

Analyses of the outcomes were conducted by IBM SPSS Statistics, version 23.0 (2010 SPSS Inc., IBM Company, Armonk, NY, USA). Descriptive statistics (percentages, means, and standard deviations [SD]) were used to summarize baseline participant characteristics and scores on the self-report measures. Chi-square and Student's $t$ tests were performed to test differences of between-group. The repeated-measures ANOVA models were conducted to analyze the changes from baseline in the outcome measures. Median and interquartile intervals were used to describe data of non-normal distribution (Satisfaction) and the Wilcoxon rank-sum test was used for comparisons between groups. All of the tests were two-sided, and statistical significance was set at $p<0.05$.

\section{Results}

\section{Sample characteristics}

One hundred twenty patients were interested in participation and assessed eligibility. Following inclusion and exclusion criteria, finally 100 patients were approached for the study. Figure 1 shows the CONSORT flow diagram, including information about study exclusion and the sample size for analysis. Table 1 summarizes the demographic data that participants were well-matched 


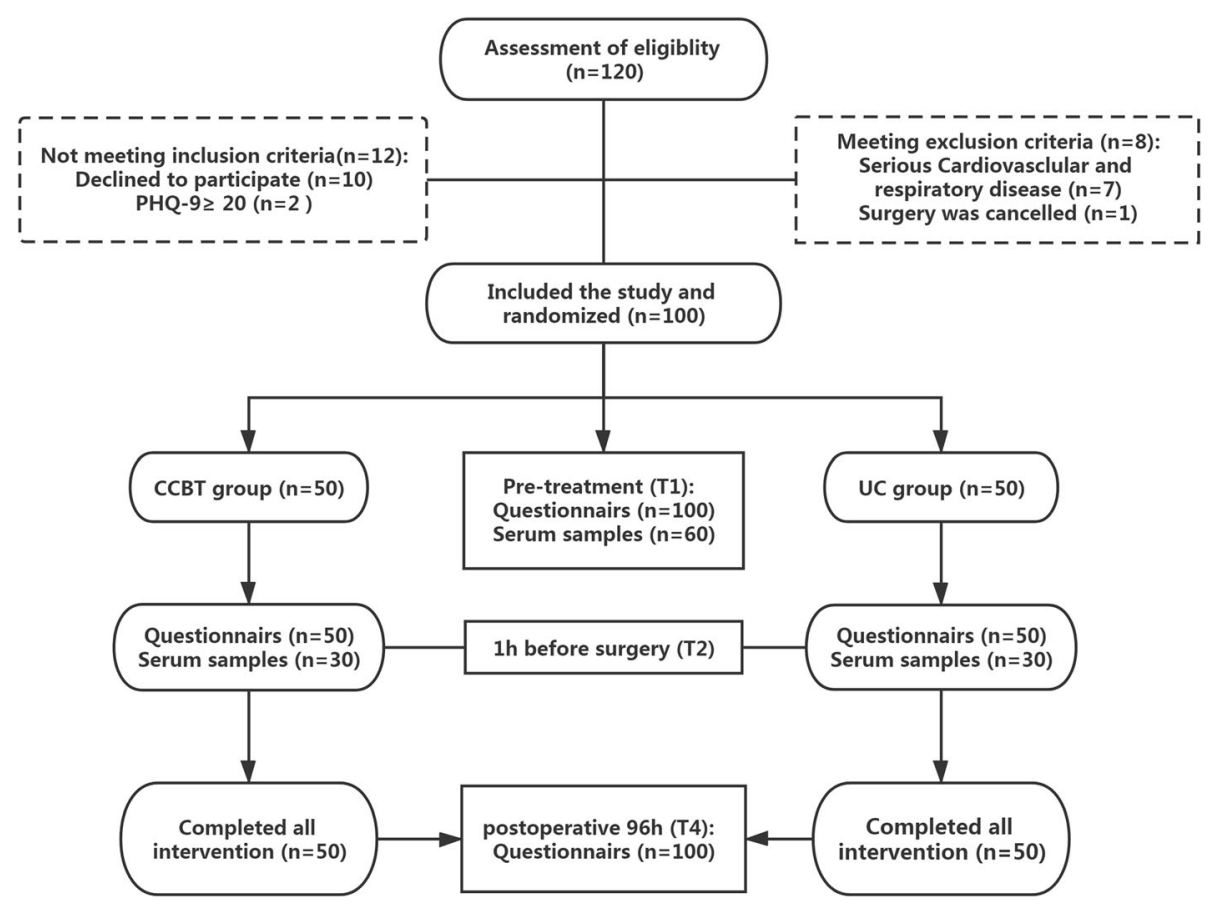

Fig. 1 CONSORT flow diagram

in terms of baseline characteristics between the two groups.

\section{Comparative analysis between two groups on the SAI, PHQ-9, and AIS scores}

The results of repeated-measures ANOVA found group*time interaction and time effect for SAI and AIS scores was statistically significant (all $p<0.01$ ). Further simple effect analysis found, to compare with UC group,

Table 1 Characteristics of the study population

\begin{tabular}{llll}
\hline Variable & $\begin{array}{l}\text { UC group } \\
(\boldsymbol{n}=\mathbf{5 0})\end{array}$ & $\begin{array}{l}\text { CCBT group } \\
(\boldsymbol{n}=\mathbf{5 0})\end{array}$ & $\boldsymbol{p}$ \\
\hline Age (mean \pm SD) & $43.08 \pm 12.51$ & $42.10 \pm 12.89$ & 0.70 \\
Gender (\%) & $25(50.00)$ & $22(44.00)$ & 0.55 \\
$\quad$ Men & $25(50.00)$ & $28(56.00)$ & \\
$\quad$ Women & & & \\
Education (\%) & $36(72.00)$ & $37(74.00)$ & 0.82 \\
$\quad$ High school graduate & $14(28.00)$ & $13(26.00)$ & \\
$\quad$ Non-graduates & $34.50 \pm 8.01$ & $35.18 \pm 9.51$ & 0.87 \\
$\quad$ TAl (mean \pm SD) & & & \\
Diagnosis (\%) & $26(52.00)$ & $30(60.00)$ & 0.574 \\
CRS with NP & $20(40.00)$ & $15(30.00)$ & \\
CRS (only) & $4(8.00)$ & $5(10.00)$ & \\
Other & & & \\
\hline
\end{tabular}

All values are mean \pm SD or number (proportion). TAl Spielberger Trait Anxiety Inventory. CRS with NP chronic rhinosinusitis with nasal polyps, CRS chronic rhinosinusitis the mean scores of SAI in CCBT group were lower and had a difference value $5.04(95 \% \mathrm{CI}-7.50,-2.54)$ and 5.64 (95\% CI - 8.01, - 3.02) at T2 and T3 (all $p<0.01$ ). The mean scores of AIS in CCBT group were lower and had a difference value $1.26(95 \% \mathrm{CI}-2.05,-0.47)$ and 1.26 (95\% CI $-2.09,-0.50)$ at T3 and T4 (all $p<0.01$ ). For PHQ-9 scores, group*time interaction was not statistically significant $(p=0.06)$ and time effect was statistically significant $(p<0.01)$ (see Table 2$)$.

\section{The comparison about the positive rate of participants}

The results of the comparison about positive rate of participants who were moderate and severe anxiety (SAI score > 37) illustrated that the difference was statistically significant between the two group ( $72 \%$ vs. $88 \%, p=0.04)$. The results of the comparison about positive rate of participants who were insomnia (AIS score $>5$ ) illustrated that the difference after intervention was statistically significant between the two groups ( $4 \%$ vs $22 \%, p=0.01$ ), while the results show no difference about the positive rate for depression (PHQ-9 $>4$ ) between two groups (Fig. 2).

The comparison about duration of surgery, anesthesia, and PACU between-two groups

The duration of surgery between-two groups had no difference significantly $(p=0.583)$. The duration of anesthesia and PACU in the CCBT group were shorter than that of the UC group $(p=0.001$ and $p<0.001)$ (see Table 3). 
Table 2 Between-group differences on study outcome measures

\begin{tabular}{|c|c|c|c|c|}
\hline Measure & UC group $(n=50)$ & CCBT group $(n=50)$ & Difference $(95 \% \mathrm{Cl})$ & $p^{d}$ \\
\hline \multicolumn{5}{|l|}{ SAI } \\
\hline $\mathrm{T} 1$ & $32.02 \pm 5.89$ & $33.12 \pm 6.80$ & $1.10(-1.35,3.61)$ & 0.39 \\
\hline $\mathrm{T} 2$ & $44.80 \pm 7.06^{\mathrm{e}}$ & $39.76 \pm 4.95^{e}$ & $-5.04(-7.04,-2.63)$ & $<0.01$ \\
\hline T4 & $28.98 \pm 5.47^{e, f}$ & $23.34 \pm 3.37^{e, f}$ & $-5.64(-7.44,-3.84)$ & $<0.01$ \\
\hline$p$ & $<0.01^{\mathrm{a}}$ & $<0.01^{\mathrm{b}}$ & $<0.01^{c}$ & \\
\hline \multicolumn{5}{|l|}{ PHQ-9 } \\
\hline $\mathrm{T} 1$ & $3.40 \pm 3.26$ & $3.14 \pm 2.48$ & $-0.26(-1.32,0.92)$ & 0.66 \\
\hline T3 & $3.98 \pm 2.91$ & $4.18 \pm 2.50$ & $-0.20(-1.26,0.98)$ & 0.71 \\
\hline T4 & $2.08 \pm 1.90$ & $1.98 \pm 1.73$ & $0.10(-0.94,1.31)$ & 0.78 \\
\hline$p$ & $0.81^{\mathrm{a}}$ & $<0.01^{b}$ & $0.06^{c}$ & \\
\hline \multicolumn{5}{|l|}{ AIS } \\
\hline $\mathrm{T} 1$ & $3.16 \pm 2.44$ & $3.60 \pm 2.16$ & $0.44(-0.47,1.34)$ & 0.91 \\
\hline T3 & $6.16 \pm 2.21^{e}$ & $4.90 \pm 1.73^{\mathrm{e}}$ & $-1.26(-2.05,-0.47)$ & 0.002 \\
\hline T4 & $3.18 \pm 2.22^{9}$ & $1.92 \pm 1.86^{\mathrm{e}, \mathrm{g}}$ & $-1.26(-2.07,-0.45)$ & 0.003 \\
\hline$p$ & $0.005^{a}$ & $<0.01^{b}$ & $<0.01^{\mathrm{C}}$ & \\
\hline
\end{tabular}

SAI State Anxiety Inventory, PHQ-9 Patient Health Questionnaire Depression Scale-9 item, AIS Athens Insomnia Scale, T1 before the intervention, $T 2$ at $1 \mathrm{~h}$ before surgery, $T 3$ at postoperative $48 \mathrm{~h}, \mathrm{T4}$ at postoperative $96 \mathrm{~h}$ (after the intervention completed)

${ }^{a} p$ value of group effect

${ }^{b} p$ value of time effect

${ }^{c} p$ value of interactive effects between time and group

${ }^{d} p$ value adjusted by Bonferroni method

${ }^{\mathrm{e}}$ Compared with the T1, $p<0.05$, adjusted by Bonferroni method

${ }^{f}$ Compared with the T2, $p<0.05$, adjusted by Bonferroni method

${ }^{9}$ Compared with the T3, $p<0.05$, adjusted by Bonferroni method

The comparison of Cortisol and ACTH levels between-two groups

The cortisol level of the UC group were significantly greater at $\mathrm{T} 2$ compared to $\mathrm{T} 1(p=0.001)$. At $\mathrm{T} 2$, the cortisol level were higher significantly in the UC group than that of the CCBT group $(p=0.02)$ (see Table 4$)$.

The change trend comparison about SBP, DBP, and HR The change trend comparison about SBP, DBP, and HR between two groups found that the trend for CCBT group was more stabilized than the UC group. More importantly, at $1 \mathrm{~h}$ before surgery, the SBP mean value in CCBT group was lower than that of the UC group $(127.24 \pm 16.27$ vs $146.06 \pm 20.59, p<0.01)$, the same with the DBP mean value $(78.48 \pm 11.42$ vs $87.86 \pm$ $13.31, p<0.01)$ and the HR mean value $(79.90 \pm 10.73$ vs $90.42 \pm 12.83, p<0.01$ ) (Fig. 3).

\section{Satisfaction survey comparisons between two groups}

Participant satisfaction was significantly higher in the CCBT group than in the UC group for all of the assessed categories except disease care $(p=0.12)$ before discharge care item (Table 5).

\section{Discussion}

In the present study, we aimed to explore the efficacy of an optimized five-sessions cCBT for individuals with FESS who had psychobiological responses because of

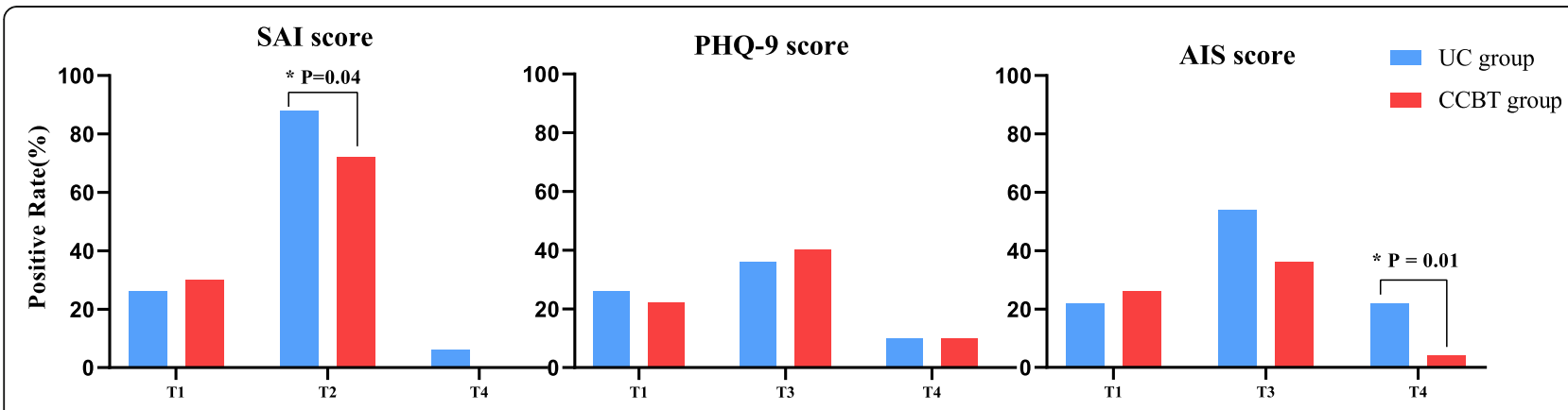

Fig. 2 The comparison about the positive rate of participants 
Table 3 The duration comparative analysis between two groups(mean \pm SD)

\begin{tabular}{llll}
\hline Duration $(\mathbf{m i n})$ & UC group $(\boldsymbol{n}=\mathbf{5 0})$ & CCBT group $(\boldsymbol{n}=\mathbf{5 0})$ & $\boldsymbol{p}$ \\
\hline Surgery & $137.78 \pm 18.41$ & $139.80 \pm 18.25$ & 0.58 \\
Anesthesia & $214.68 \pm 20.55$ & $199.24 \pm 22.79$ & 0.001 \\
PACU & $32.20 \pm 8.84$ & $22.98 \pm 8.26$ & $<0.01$ \\
\hline PACU post-anesthesia care unit, Duration of anesthesia time between the induction and leaving from PACU, Duration of surgery time between touching the skin \\
and the end of suture or tamponade, Duration of PACU time between PACU admission to discharge
\end{tabular}

surgery. The intervention appeared to be useful not only in improving anxiety and insomnia, but also in biological parameters (cortisol, hemodynamic control) compared to the control group. However, there were few RCTs reported about CCBT treating FESS patients with psychological distress. The present study provides preliminary data to assess the potential efficacy and mechanism of mobile technology-based cCBT interventions in clinical surgery applications.

In the present study, the anxiety level increased before surgery in both groups. The causes may be the lack of an adequate preoperative education, awareness of the process of surgery and anesthesia, and uncertainties about postoperative steps and worrying about the outcomes. The newly cCBT based on nursing education can help patients to know surgery and anesthesia adequately, and preview postoperative rehabilitation in advance. The result showed the CCBT group experienced a lower level of anxiety and insomnia than the control group. The improvement in insomnia may be affected by the improvement of anxiety. The results were consistent with the results of recent meta-analyses, and $\mathrm{CCBT}$ was found to improve self-reported psychological outcomes (anxiety and depression) and physical outcomes (pain and insomnia) of patients with psychiatric and somatic disorders (Mehta et al. 2019). However, no significant effects were observed for depression symptoms between the two groups. It may be due to surgery-related stress being experienced on a short-term timescale, while depression may exhibit a more extended time course (Yang et al., 2015). The mechanisms that psychological interventions can influence anxiety in surgery patients may be affected underlying the neuroendocrine response to surgical stress (Villa et al. 2020).

The surgery-related perioperative stress may affect extensively patients' neuroendocrine pathways (Maduka et al. 2015). Enhanced cognitive coping may modulate hypothalamic-pituitary-adrenal (HPA) axis activity and improve the levels of endocrine hormones (Abelson et al. 2008). Anxiety can increase the cortisol secretion, which has been observed among patients awaiting operation. The newly developed CCBT based on CBT and nursing education can significantly reduce cortisol levels of the CCBT group, which may be due to cognitive bias modification and behavioral coping in CBT. Cortisol homeostasis is essential for cognitive and affective functions (O'Connor et al. 2021). Increased cortisol and an imbalance of the HPA axis can contribute to the development of anxiety and depression (van Dalfsen and Markus, 2018). The differences of ACTH were not observed and it may illustrate that ACTH is not a sensitive index related-surgery stress than cortisol. The connection among emotions, hormones, and brain networks is the basis for subsequent research.

Numerous studies and meta-analyses have linked BP responses to mental stress to poor health outcomes (O'Connor et al. 2021). Generally, anxiety increases blood pressure, systemic vascular resistance, sympathetic activity (Pan et al. 2015). Preoperative stable hemodynamics contributes to control hypotension and reduce intraoperative bleeding which help to complete the surgery smoothly and lighten the burden on the anesthesiologist. Gu et al. in their study relieved anxiety and stabilized BP and HR by dexmedetomidine combined with parecoxib before anesthesia induction in patients for FESS (Gu et al., 2020). In this study, the patients in the CCBT group had a lower preoperative BP and $\mathrm{HR}$, which showed CCBT can help anesthesiologists control high $\mathrm{BP}$ and $\mathrm{HR}$ using minimal drugs. The shorter duration of anesthesia and PACU illustrated patients using CCBT program experienced shorter duration of anesthesia induction and faster postoperative recovery time. These are key factors of improved perioperative outcomes and can also ease the burden for anesthetists

Table 4 Cortisol and ACTH comparative analysis between two groups(mean \pm SD)

\begin{tabular}{llllll}
\hline Measure & \multicolumn{2}{l}{ UC $(\boldsymbol{n}=\mathbf{3 0})$} & & CCBT $(\boldsymbol{n}=\mathbf{3 0})$ & T2 \\
\cline { 2 - 3 } & T1 & T2 & & T1 & $306.22 \pm 74.86$ \\
\hline Cortisol $(\mathrm{nmol} / \mathrm{L})$ & $295.43 \pm 125.66$ & $349.57 \pm 83.39 \Delta^{*}$ & & $307.07 \pm 111.92$ & $39.55 \pm 20.30$ \\
ACTH $(\mathrm{pg} / \mathrm{mL})$ & $38.17 \pm 17.40$ & $40.57 \pm 13.41$ & & $37.39 \pm 24.70$ & 39.55 \\
\hline
\end{tabular}

$T 1$ before the intervention, $T 2$ at $1 \mathrm{~h}$ before surgery

${ }^{*} p<0.05, p$ value of independent-samples $t$ test

$\Delta p<0.05, p$ value of paired t test 

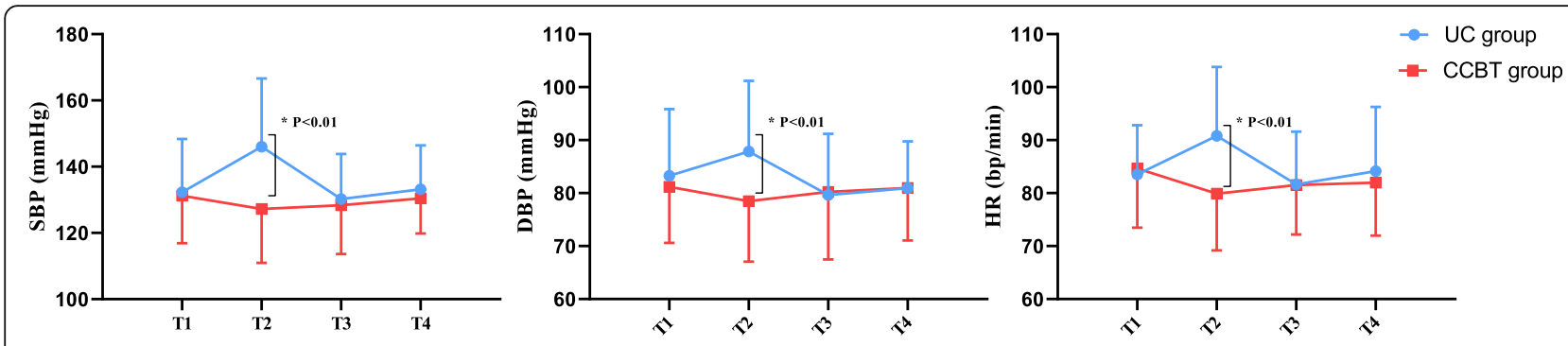

Fig. 3 The change trend line chart of $B P$ and $H R$

(Sgrò et al. 2019), while the duration of surgery was no difference between two groups, which may be related to different surgeons. In the present study, cCBT had a positive effect on stabilizing hemodynamics before anesthesia induction, but had a limited impact on vital signs during the postoperative recovery period.

Making the patient safe and comfortable during the whole perioperative period is our medical aim. Satisfaction scores were universally high in the CCBT group. However, differences in Disease Care item was not observed, which may be correlated to accept standard nursing procedures. The patients participating in the CCBT group indicated that they received more services, especially psychological services. The more satisfactory medical experience makes the relationship between medical staff and patients harmonious and reduces the incidence of violence (Meng et al. 2020).

The study had several limitations. Firstly, the intervention was conducted at a single site, with a relatively small sample size, and only a part of participants were willing to be blood collected, which may have been insufficient and subject to type II errors during analysis. Secondly, our study did not assess the long-term effects in patients, which can be rectified in a future study that continues to follow up on these patients and collect additional data. Thirdly, we did not measure intraop blood loss or surgeon's satisfaction of a bloodless field, which are important measures to evaluate the success of FESS. Two teams of surgeons may also introduce heterogeneity because of different experience. Finally, further research direction will classify different populations and optimize the $\mathrm{CCBT}$ program to become more personalized and targeted.

Table 5 Satisfaction comparison between two groups before discharge, median $\left(\mathrm{P}_{25}, \mathrm{P}_{75}\right)$

\begin{tabular}{llll}
\hline Item & $\begin{array}{l}\text { UC group } \\
(\boldsymbol{n}=\mathbf{5 0})\end{array}$ & $\begin{array}{l}\text { CCBT group } \\
(\boldsymbol{n}=\mathbf{5 0})\end{array}$ & $\boldsymbol{p}$ \\
\hline Psychological care & $8(7,10)$ & $10(9,10)$ & $<0.01$ \\
Disease care & $20(18,20)$ & $20(18,20)$ & 0.12 \\
Psychosomatic management & $15(14,17.5)$ & $20(16.5,20)$ & $<0.01$ \\
Total & $43(40,45.5)$ & $48(45,50)$ & $<0.01$ \\
\hline
\end{tabular}

\section{Conclusion}

In conclusion, we found that the optimized cCBT program may be a suitable and effective adjunctive treatment relieving psychobiological responses for patients undergoing FESS. With the help of multidisciplinary treatment, the new form of psychosomatic intervention can improve the access to psychological services and surgical outcomes.

\section{Abbreviations}

FESS: Functional endoscopic sinus surgery; CRS: Chronic rhino sinusitis; TIVA: Total intravenous anesthesia; CBT: Cognitive behavior therapy; CCBT: Computer-assisted cognitive-behavior therapy; UC: Usual-care; ERAS: Enhanced recovery after surgery; SAl: State anxiety inventory; TAI: Trait anxiety inventory; PHQ-9: Patients health questionnaire depression scale-9 item; AIS: Athens insomnia scale; ACTH: Adrenocorticotropic hormone; HPA: Hypothalamic-pituitary-adrenal; CLIA: Chemiluminescence assay; SBP: Systolic blood pressure; DBP: Diastolic blood pressure; HR: Heart rate; PACU: Post-anesthesia care unit

\section{Supplementary Information}

The online version contains supplementary material available at https://doi. org/10.1186/s13741-021-00195-3.

Additional file 1: Supplementary Table 1. State Trait Anxiety Scale (STAI). Supplementary Table 2. Patients Health Questionnaire-9 (PHQ-9). Supplementary Table 3. Athens Insomnia Scale (AIS). Supplementary Table 4. Satisfaction Survey Questionnaire.

\section{Acknowledgements}

We would like to thank all participants who made this study possible and all staff who participated sufficiently in the work. For the data analysis, we thank Shanxi Hengtai of Yifeng Science and Technology Development CO., LTD.

\section{Authors' contributions}

$Y Y, Y L L, Y X$, and BQW designed this study protocol. YY and HBZ collected, managed and analyzed data. YY drafted the manuscript. YLL, YX, and BQW revised the manuscript. All authors contributed to initial and final drafts. The authors read and approved the final manuscript.

\section{Funding}

This study was supported by the 136 Medical Rejuvenation Project of Shanxi Province, the health research project of Health Commission of Shanxi

Province (2018046) and the soft science research plan project of the Shanxi Science and Technology Department (2018041029-6). The funding had no further role in the study design; in the collection, analysis, and interpretation of data; in the writing of the report; and the decision to submit the paper for publication.

\section{Availability of data and materials}

The dataset used and analyzed during the current study is available from the corresponding author on reasonable request. 


\section{Declarations}

\section{Ethics approval and consent to participate}

The study had been performed in accordance with the Declaration of Helsinki. The study protocol was conducted with the consent of the Ethics Committee of First Hospital of Shanxi Medical University in China on July 31, 2019 (document number [2019]K-SK028). The informed consents to participate in the study had been obtained from participants.

\section{Competing interests}

The authors declare that they have no competing interests.

\section{Author details}

'Nursing College, Shanxi Medical University, Taiyuan, People's Republic of China. ${ }^{2}$ Nursing Department, The First Hospital of Shanxi Medical University, Taiyuan, People's Republic of China. ${ }^{3}$ Department of Anesthesia, The First Hospital of Shanxi Medical University, Taiyuan, People's Republic of China. ${ }^{4}$ Department of Psychiatry, The First Hospital of Shanxi Medical University, Taiyuan, People's Republic of China. ${ }^{5}$ Shanxi Key Laboratory of Artificial Intelligence Assisted Diagnosis and Treatment for Mental Disorder, The First Hospital of Shanxi Medical University, Taiyuan, People's Republic of China. ${ }^{6}$ Department of Otorhinolaryngology Head and Neck Surgery, The First Hospital of Shanxi Medical University, Taiyuan, People's Republic of China. ${ }^{7}$ Shanxi Key Laboratory of Otorhinolaryngology Head and Neck Cancer, The First Hospital of Shanxi Medical University, Taiyuan, Shanxi, People's Republic of China.

Received: 2 October 2020 Accepted: 31 May 2021

Published online: 19 August 2021

\section{References}

Abelson J, Khan S, Liberzon I, Erickson T, Young E. Effects of perceived control and cognitive coping on endocrine stress responses to pharmacological activation. Biol Psychiatry. 2008;64(8):701-7. https://doi.org/10.1016/j. biopsych.2008.05.007.

Andrews G, Basu A, Cuijpers P, Craske M, McEvoy P, English C, et al. Computer therapy for the anxiety and depression disorders is effective, acceptable and practical health care: an updated meta-analysis. J Anxiety Disord. 2018;55:708. https://doi.org/10.1016/j.janxdis.2018.01.001

Beck A. Thinking and depression: II. Theory and Therapy. Arch Gen Psychiatry. 1964;10(6):561-71. https://doi.org/10.1001/archpsyc.1964.0172 0240015003.

Carlbring P, Andersson G, Cuijpers P, Riper H, Hedman-Lagerlöf E. Internet-based vs. face-to-face cognitive behavior therapy for psychiatric and somatic disorders: an updated systematic review and meta-analysis. Cogn Behav Ther 2018:47(1):1-18. https://doi.org/10.1080/16506073.2017.1401115.

Cartreine J, Ahern D, Locke S. A roadmap to computer-based psychotherapy in the United States. Harv Rev Psychiatry. 2010;18(2):80-95. https://doi.org/10.31 09/10673221003707702

Chen Bing, C.Y., Dong Hailong etc., 2018. Chinese consensus and clinical guidelines for Enhanced Recovery After Surgery (2018 editon). Chin J Pract Surg v.38 (01), 1-20.

Chhabra A, Saini P, Sharma K, Chaudhary N, Singh A, Gupta S. Controlled hypotension for FESS: A randomised double-blinded comparison of magnesium sulphate and dexmedetomidine. Indian J Anaesth. 2020;64(1): 24-30. https://doi.org/10.4103/ija.IJA_417_19.

Dao TK, Youssef NA, Armsworth M, Wear E, Papathopoulos KN, Gopaldas R. Randomized controlled trial of brief cognitive behavioral intervention for depression and anxiety symptoms preoperatively in patients undergoing coronary artery bypass graft surgery. J Thorac Cardiovasc Surg. 2011;142(3): $109-15$.

Epel E. Can childhood adversity affect telomeres of the next generation? Possible mechanisms, implications, and next-generation research. Am J Psychiatry. 2020;177(1):7-9. https://doi.org/10.1176/appi.ajp.2019.19111161.

Gu X, Tan X, Chen J, Wang J, Lu Y, Zhang L. The clinical effect of dexmedetomidine combined with parecoxib sodium on sedation, antianxiety and prevention of intubation stress in patients undergoing functional endoscopic sinus surgery: a randomised controlled trial. BMC Anesthesiol. 2020;20(1):166. https://doi.org/10.1186/s12871-020-01080-0.

Kane $\mathrm{K}$. The early history and development of functional endoscopic sinus surgery. J Laryngol Otol. 2020;134(1):8-13. https://doi.org/10.1017/S0022215119002457.
Kaper N, van der Heijden G, Cuijpers S, Stokroos R, Aarts M. A comparison of international clinical practice guidelines on adult chronic rhinosinusitis shows considerable variability of recommendations for diagnosis and treatment. Eur Arch Otorhinolaryngol. 2020;277(3):659-68.

Kroenke K, Spitzer RL, Williams JBW. The PHQ-9 : validity of a brief depression severity measure. J Gen Intern Med. 2001;16(9):606-13. https://doi.org/10.104 6/j.1525-1497.2001.016009606.x.

Landon L, Douglas G, Downs M, Greene M, Whitmire A, Zwart S, et al. The Behavioral biology of teams: multidisciplinary contributions to social dynamics in isolated, confined, and extreme environments. Front Psychol. 2019;10:2571. https://doi.org/10.3389/fpsyg.2019.02571.

Lu V, Phan K, Oh L. Total intravenous versus inhalational anesthesia in endoscopic sinus surgery: A meta-analysis. Laryngoscope. 2020;130(3):575-83. https://doi.org/10.1002/lary.28046.

Maduka I, Neboh E, Ufelle S. The relationship between serum cortisol, adrenaline, blood glucose and lipid profile of undergraduate students under examination stress. Afr Health Sci. 2015;15(1):131-6. https://doi.org/10.4314/ahs.v15i1.18.

Martin G, Kaufman D, Marik P, Shapiro N, Levett D, Whittle J, et al. Perioperative Quality Initiative (POQI) consensus statement on fundamental concepts in perioperative fluid management: fluid responsiveness and venous capacitance. Perioperative Med. 2020;9:12.

Mehta S, Peynenburg V, Hadjistavropoulos H. Internet-delivered cognitive behaviour therapy for chronic health conditions: a systematic review and meta-analysis. J Behav Med. 2019;42(2):169-87. https://doi.org/10.1007/s1 0865-018-9984- $x$

Meng Y, Li Y, Cao H, Xu Y, Wang B. Development of two psychological experience questionnaires for screening violence-related mental health disorders of non-psychiatric inpatients. Health Qual Outcomes. 2020;18(1): 151. https://doi.org/10.1186/s12955-020-01399-9.

Modir H, Modir A, Rezaei O, Mohammadbeigi A. Comparing remifentanil, magnesium sulfate, and dexmedetomidine for intraoperative hypotension and bleeding and postoperative recovery in endoscopic sinus surgery and tympanomastoidectomy. Med Gas Res. 2018;8(2):42-7. https://doi.org/10.41 03/2045-9912.235124.

O'Connor D, Thayer J, Vedhara K. Stress and health: a review of psychobiological processes. Ann Rev Psychol. 2021;72(1):663-88. https://doi.org/10.1146/a nnurev-psych-062520-122331.

Pan X, Zan W, Xiong M, al., e. Related risk factors of moderate and severe preoperative anxiety. J Clin Anesthesiol. 2018;5(34):425-8.

Pan Y, Cai W, Cheng Q, Dong W, An T, Yan J. Association between anxiety and hypertension: a systematic review and meta-analysis of epidemiological studies. Neuropsychiatr Dis Treat. 2015;11:1121-30. https://doi.org/10.2147/ NDT.S77710.

Ruffinengo $C$, Versino E, Renga G. Effectiveness of an informative video on reducing anxiety levels in patients undergoing elective coronarography: an RCT. Eur J Cardiovasc Nurs. 2009;8(1):57-61. https://doi.org/10.1016/j. ejcnurse.2008.04.002.

Sgrò S, Morini F, Bozza P, Piersigilli F, Bagolan P, Picardo S. Intravenous propofol allows fast intubation in neonates and young infants undergoing major surgery. Front Pediatr. 2019;7:321. https://doi.org/10.33 89/fped.2019.00321.

Shi J, Fu Q, Zhang H, Cheng L, Wang Y, Zhu D, et al. Epidemiology of chronic rhinosinusitis: results from a cross-sectional survey in seven Chinese cities. Allergy. 2015;70(5):533-9. https://doi.org/10.1111/all.12577.

Soldatos CR, Dikeos DG, Paparrigopoulos TJ. Athens Insomnia Scale: validation of an instrument based on ICD-10 criteria. J Psychosom Res. 2000;48(6):555-60. https://doi.org/10.1016/S0022-3999(00)00095-7.

Soldatos CR, Dikeos DG, Paparrigopoulos TJ. The diagnostic validity of the Athens Insomnia Scale. J Psychosom Res. 2003:55(3):260-7.

Spielberger CD, Gorsuch RL, Lushene RE, Vagg PR, Jacobs GA. Manual for the state-trait anxiety inventory (Form Y1 - Y2). Palo Alto, CA: Consulting Psychogyists Press; 1983.

Sun $Y$, Li Y, Sun $Y$, Wang $X$, Ye $H$, Yuan $X$. Dexmedetomidine effect on emergence agitation and delirium in children undergoing laparoscopic hernia repair: a preliminary study. J Int Med Res. 2017;45(3):973-83. https:// doi.org/10.1177/0300060517699467.

van Dalfsen J, Markus C. The influence of sleep on human hypothalamicpituitary-adrenal (HPA) axis reactivity: A systematic review. Sleep Med Rev. 2018;39:187-94. https://doi.org/10.1016/j.smrv.2017.10.002.

Villa G, Lanini I, Amass T, Bocciero V Scirè Calabrisotto C, Chelazzi C, et al. Effects of psychological interventions on anxiety and pain in patients undergoing 
major elective abdominal surgery: a systematic review. Perioper Med. 2020; 9(1):38.

Visioni A, Shah R, Gabriel E, Attwood K, Kukar M, Nurkin S. Enhanced recovery after surgery for noncolorectal surgery?: a systematic review and metaanalysis of major abdominal surgery. Ann Surg. 2018;267(1):57-65. https://doi. org/10.1097/SLA.0000000000002267.

Wright J, McCray L, Eells T, Gopalraj R, Bishop L. Computer-assisted cognitivebehavior therapy in medical care settings. Curr Psychiatry Rep. 2018;20(10):92. https://doi.org/10.1007/s11920-018-0947-2.

Yang PL, Huang GS, Tsai CS, Lou MF. Sleep quality and emotional correlates in Taiwanese coronary artery bypass graft patients 1 week and 1 month after hospital discharge: a repeated descriptive correlational study. Plos one. 2015;10(8):e0136431.

\section{Publisher's Note}

Springer Nature remains neutral with regard to jurisdictional claims in published maps and institutional affiliations.

Ready to submit your research? Choose BMC and benefit from:

- fast, convenient online submission

- thorough peer review by experienced researchers in your field

- rapid publication on acceptance

- support for research data, including large and complex data types

- gold Open Access which fosters wider collaboration and increased citations

- maximum visibility for your research: over $100 \mathrm{M}$ website views per year

At BMC, research is always in progress.

Learn more biomedcentral.com/submissions 